\title{
Contextual Effects on the Perceived Health Benefits of Exercise: The Exercise Rank Hypothesis
}

\author{
John Maltby, ${ }^{1}$ Alex M. Wood, ${ }^{2}$ Ivo Vlaev, ${ }^{3}$ \\ Michael J. Taylor, ${ }^{3}$ and Gordon D.A. Brown ${ }^{4}$ \\ ${ }^{1}$ University of Leicester; ${ }^{2}$ University of Stirling; \\ ${ }^{3}$ Imperial College London; ${ }^{4}$ University of Warwick
}

\begin{abstract}
Many accounts of social influences on exercise participation describe how people compare their behaviors to those of others. We develop and test a novel hypothesis, the exercise rank hypothesis, of how this comparison can occur. The exercise rank hypothesis, derived from evolutionary theory and the decision by sampling model of judgment, suggests that individuals' perceptions of the health benefits of exercise are influenced by how individuals believe the amount of exercise ranks in comparison with other people's amounts of exercise. Study 1 demonstrated that individuals' perceptions of the health benefits of their own current exercise amounts were as predicted by the exercise rank hypothesis. Study 2 demonstrated that the perceptions of the health benefits of an amount of exercise can be manipulated by experimentally changing the ranked position of the amount within a comparison context. The discussion focuses on how social norm-based interventions could benefit from using rank information.
\end{abstract}

Keywords: social, norm, influence, evolutionary, judgment, decision making

According to the WHO Global Strategy Report on Diet, Physical Activity, and Health, exercise is important to the healthy development of children and young people, and continued patterns of regular exercise reduce the risk of developing cardiovascular disease, diabetes, colon cancer, and breast cancer in later life (World Health Organization, 2006). The report identifies children and young people as needing special attention, so that exercise habits are developed early in life, with formal recommendations suggesting $1 \mathrm{hr}$ or more of moderate-intensity physical activity on five or more days per week. There are concerns, however, that only one third of adolescents reach this target and that individuals may be at risk for adverse health outcomes later in life due to not developing adequate exercise

John Maltby is with the College of Medicine, Biological Sciences and Psychology, University of Leicester, Leicester, UK. Alex M. Wood is with the Stirling Management School, University of Stirling, Stirling, UK. Ivo Vlaev and Michael J. Taylor are with the Division of Surgery, Imperial College London, London, UK. Gordon D.A. Brown is with the Department of Psychology, University of Warwick, Coventry, UK. 
habits in childhood and early adulthood (Currie et al., 2006). Consequently, it is important to understand how individuals perceive the health benefits associated with their own amount of exercise, not least because such an understanding may inform the development of strategies and interventions for promoting exercise that are consistent with people's natural way of processing information.

Many theories of how individuals make judgments around exercise suggest that individuals determine, or judge the acceptability of, their own levels of exercise at least partly by reference to what other individuals do (Carron, Hausenblas \& Mack, 1996). For example, the organismic integration approach within self-determination theory suggests that the behavior or motivations endorsed by significant others can be internalized by an individual (Deci \& Ryan, 1985). Other theories emphasize the importance of normative influence, which may occur via the influence of subjective norms (i.e., the theory of planned behavior [Ajzen, 1991]), descriptive norms (i.e., the focus theory of normative conduct; [Cialdini, Kallgren, \& Reno, 1991]), or prototypes (the prototype-willingness model [Gibbons, Gerrard, Blanton, \& Russell, 1998]).

These accounts of how individuals compare their level of exercise to that of others have typically suggested that people compare their own amount of exercise to a single average, reference point, or aggregate assessment based on the prevalence or incidence of other individuals' exercise amounts. To illustrate, a typical assessment of subjective norm influence (or normative expectancies) might involve examining an individual's consideration of (1) whether a particular group of individuals engage in exercise (e.g., "do particular referents engage in exercise?"), or (2) a value derived from the frequency of exercise among a relevant social group (e.g., "30 min" or "four or more times per week"; Rhodes, Blanchard, Courneya, $\&$ Plotnikoff, 2009). The measurement of descriptive norm influence also typically relies on a single measure of physical activity (e.g., the average or aggregate) that describes the amount of exercise carried out by specific groups (e.g., friends, coworkers; Priebe \& Spink, 2011). When assessing the influence of prototypes, respondents are typically asked to compare their exercise behavior to images of an exercise social group prototype (e.g., a "typical" person who exercises; Ouellette, Hessling, Gibbons, Reis-Bergan, \& Gerrard, 2005).

It is important to understand exactly how individuals compare their own amount of exercise with that of others to inform the development of interventions to encourage exercise. For example, within the public health literature on exercise, individuals are typically asked to compare their own activity against a single figure, such as the Centers for Disease Control and Prevention recommendation of $150 \mathrm{~min}$ a week of moderate-intensity aerobic activity to lower the risk of cardiovascular disease (Centers for Disease Control and Prevention, 2011). However, messages may not produce the greatest possible change in the desired attitude or behavior if they do not target people's natural ways of making judgments.

Here we develop and test a specific hypothesis of how individuals socially compare when making judgments about exercise levels. We propose an exercise rank hypothesis, according to which people make judgments about amounts of exercise based on the ranked position of those amounts within a social context. To illustrate, two people may exercise for the same amount of time each week, but the person who perceives their amount of exercise to rank higher among other people's amounts might imagine themselves to be gaining greater health benefits. 
We integrate two theoretical approaches: (1) evolutionary theories of rank sensitivity and (2) the decision by sampling (DbS) model of judgment and decision making (Stewart, Chater, \& Brown, 2006) from cognitive psychology. Evolutionary theory suggests a possible motivation for why individuals' judgments about exercise may be influenced by their perception of their ranked position within a social group. The DbS model provides an explanation of the cognitive processes that are associated with social ranking and which underpin the judgments made about one's own amount of exercise in relation to others.

Evolutionary theory suggests that within higher mammals' social systems there is a strong motivation for members to compare themselves against one another to determine social rank. Such comparisons lead to the formation of group hierarchies, which influence behavior (Sapolsky, 2004, Wilson, 1975). Individuals of a higher social rank achieve a dominant breeding position, in terms of both intersexual and intrasexual competition (e.g., attracting potential mates or overcoming rivals) and have higher chances of reproducing and ensuring the survival of themselves and their offspring (Sapolsky, 2004). In contrast, individuals of a low social rank engage in submissive, vigilant, and withdrawal behaviors, and hence low social rank may be related to avoidance (Newton, Bane, Flores, \& Greenfield, 1999; Taylor, Gooding, Wood \& Tarrier, 2011).

Moreover, ranked position within a social hierarchy is associated with the investments made by both animals and humans in their own health systems (e.g., appearance, adrenocortical, cardiovascular, dietary, reproductive, and immune systems) that influence factors such as physical growth and competition, which, in turn, influence survival and reproduction (Cummins, 1996; 2006; Sapolsky, 2004). Moreover, exercise is inextricably linked with human evolution and food procurement (Eaton et al., 2002), as it represents the physical activity that was vital in our ancestral hunter-gatherer societies (O'Keefe, Vogel, Lavie, \& Cordain, 2010; 2011). We hypothesize that engagement in exercise and the consequent physical benefits of exercise (increased physical attractiveness, healthy body weight and shape, being less likely to get ill) are markers to others of healthy physical systems, and therefore individuals who exercise are likely to be perceived as having a higher social rank. Therefore people may be biased toward evaluating people's health depending on where their exercise amount is ranked in a hierarchy, irrespective of the actual amount of time that they exercise.

Decision by sampling (Stewart et al., 2006) is a cognitive model of judgment that provides an explanation of the cognitive processes that underpin rank-based evaluations. According to $\mathrm{DbS}$ the judgment of a quantity (e.g., an amount of exercise) is context dependent and determined by the relative ranked position of the to-be-judged quantity within the context of other available quantities. We describe the model by showing how it can be applied to cognitions around exercise. The application of the DbS model to exercise cognitions proposes that, when assessing one's own amount of exercise, an individual first brings to mind a group of people, sampled either from memory or from the immediate environment. The individual then sequentially compares their own amount of exercise with the amount of exercise associated with each person in the sample, simply encoding whether each other person exercises more or less than they do themselves. The results of these comparisons are summed to give the number of people who exercise more and exercise less than the person making the judgment. The individual's rank within the retrieved distribution can 
then be calculated. For example, an individual might call to mind five people who exercise more than they do, and four who exercise less. The ranked position of that individual would then be 5 th (from the bottom), and their relative ranked position within the group would be $4 /(4+5)$. The rank-based approach embodied in the $\mathrm{DbS}$ model is consistent with a large body of experimental research showing sensitivity to rank in judgments of psychophysical (Parducci, \& Perrett, 1971; Riskey, Parducci, \& Beauchamp, 1979), personality (Wood, Brown, Maltby, \& Watkinson, 2012), health (Wood, Brown, \& Maltby, 2012), and mental health (Melrose, Brown, \& Wood, in press) variables. Thus when the DbS model is applied to judgments made around exercise, it suggests (in contrast to social influence theories that assume comparison against a single value or group norm), that individuals make multiple comparisons regarding exercise amount within the social hierarchy of a relevant norm group.

In summary, evolutionary theory, social rank, and the association of these processes with health systems suggest why rank sensitivity in judgments of exercise amounts may emerge among a population. The $\mathrm{DbS}$ model provides an explanation of the cognitive processes that underline the exercise-related judgments. Together these theories underpin our exercise rank hypothesis, which proposes that people's assessment of the health benefits they receive from exercise are influenced by multiple comparisons that determine the ranked position of an exercise amount within a context of other people's exercise amounts.

An extensive literature search did not reveal any previous study that examined whether a person's evaluation of the health benefits of their amount of exercise depends on how they perceive their exercise to rank among other individuals. Examining the exercise rank hypothesis among young adults may be particularly useful because at this age individuals are likely to be (1) engaging within competitive social hierarchies in terms of seeking and selecting mates and (2) beginning to make choices regarding exercise habits under their own volition, rather than, for example, as part of a school curriculum or following parental guidance. The current studies tested the exercise rank hypothesis. Specifically, Study 1 examined whether perceptions of health benefits from exercise among young adults could be predicted from the rank of their amount of exercise, while controlling for a number of other relevant variables including their perceived distance from a mean amount of exercise. Study 2 examined whether experimentally manipulating rank positions of exercise duration causally influenced the perception of the health benefits associated with a given amount of exercise.

\section{Study 1}

\section{Method}

Participants. One hundred twenty-two young adults (50 males, 72 females) aged between 18 and 22 years $(M=19.27, S D=1.30)$ completed a threesection questionnaire. Recruitment of participants was opportunistic, with flier advertisements displayed, after seeking relevant permissions, in 22 workplaces, community groups, and further education colleges situated in a North of England city center. Ethical approval for the study was obtained from a relevant university ethics committee and complied with the British Psychological Society Code of Ethics. Informed consent was obtained from all participants. 
Measures. Participants were asked to complete a three-section questionnaire. In Section 1, participants reported how many minutes of exercise they completed on average in a normal week. Respondents were given the definition of exercise, used by the World Health Organization (World Health Organization, 2010), as a "planned, structured, repetitive, and purposeful physical activity (e.g., brisk walking, aerobics, jogging, bicycling, swimming) in the sense that there is an objective for improvement or maintenance of one or more components of physical fitness" (p. 52).

In Section 2, participants answered three questions related to their perceptions of the health benefits of their current amount of exercise (shortened to perceived health benefits of current exercise): (1) "How healthy do you think your level of exercise is?" (scored from 1 [Very Unhealthy] to 10 [Very Healthy]), (2) "What extent of health benefits do you think you are going to get from your current level of exercise?" (scored from 1 [No Benefit] to 10 [Huge Benefit]), and (3) "How sufficient do you think your level of exercise is to avoid the serious health problems associated with low levels of exercise?" (scored from 1 [Totally Insufficient] to 10 [Totally Sufficient]). The wording of these three questions was based on the goal and objectives statements from the World Health Organization's Global Strategy on Diet, Physical Activity and Health (World Health Organization, 2004) which highlights action taken at an individual level (Question 1), awareness of the positive impact on health of physical exercise (Question 2), and reduced disease rates resulting from physical activity (Question 3). Thus the questions were designed to produce an overall measure of perceived health benefits of current exercise.

In Section 3, participants answered nine questions about their beliefs about the distribution of other young adults' average weekly amount of exercise in the UK. Each question required participants to complete the "how many" part of the question within the template: "The top $x \%$ of the UK young adult population exercise more than *how many* minutes per week on average?", with $x$ respectively taking values of $10,20,30,40,50,60,70,80$, and 90 .

\section{Results}

To demonstrate the utility of using the three questions in Section 2 as a summed measure of perceived health benefits of current exercise measure, both reliability and validity estimates were produced. Cronbach's alpha (Cronbach, 1951) produced a good internal reliability statistic of $\alpha=.87$. This is above the acceptable internal reliability criteria of .70 (Kline, 1986). To test for the structural validity (Messick, 1995) of the scale, we conducted a maximum likelihood factor analysis of responses to these three items. This analysis revealed a single factor (eigenvalue $=2.40$ ) which explained $80.03 \%$ percentage of the variance. Potential second and third factors explained only trivial additional amounts of variance $(12.80 \%$ and $7.16 \%$ respectively), with eigenvalues below one (.38 and .22 respectively). Furthermore, these factors would have explained less variance than any individual single item, with loadings of items on an unrotated first factor ranging from .75 to .92 . The existence of a single substantive factor underlying the responses to the three items was also supported with a parallel analysis of 1,000 random datasets using a 95\% cutoff point (Zwick and Velicer, 1986), in which the second eigenvalue (.32) failed to exceed the second of the three mean eigenvalues from the parallel analysis (1.15, 1.00 , and .86 ). These reliability and validity findings support the decision to form a single scale from the three items. 
Participants' own amount of exercise ranged from 0 to $700 \mathrm{~min}$ per week (median $=90.00$, interquartile range $30-180 \mathrm{~min})$ and $2.5 \%$ of respondents reported not engaging in any exercise at all. Table 1 shows the range, interquartile range and mean and median scores for estimated average amount of exercise, in minutes per week, for the top $10 \%, 50 \%$, and $90 \%$ of the UK young adult population. These average statistics demonstrate an expected decrease in the estimated average amounts of exercise from the $10 \%$ to the top $90 \%$ of the UK young adult population. However, the range statistics on this table also show that the estimates within each category vary widely between respondents, and that these estimates for one category overlap with estimates provided for other categories. These results show, that within our sample, individuals have very different perceptions about the amounts of exercise undertaken by the UK young adult population at various percentiles of the distribution.

Table 1 Range, interquartile range, and mean and median scores for estimated average amount of exercise, in minutes per week, for the top $10 \%, 50 \%$, and $90 \%$ of the UK young adult population

\begin{tabular}{ccccc}
\hline & $\begin{array}{c}\text { Range } \\
\text { (Minimum-Maximum) }\end{array}$ & $\begin{array}{c}\text { Interquartile } \\
\text { Range }\end{array}$ & Mean & Median \\
\hline $10 \%$ & $50-1600$ & $180-400$ & 333.81 & 300.00 \\
$50 \%$ & $15-700$ & $88.75-230$ & 177.16 & 120.00 \\
$90 \%$ & $0-355$ & $20-60$ & 120.00 & 30.00 \\
\hline
\end{tabular}

A two-step hierarchical multiple regression was performed to determine whether perceived rank of exercise predicts participants' perceived health benefits of current exercise after controlling for a number of variables. The first multiple regression was carried out using the data from the whole sample. In the first step, (1) sex, (2) age (as both these variables are related to both exercise and health practices; Volden, Langemo, Adamson, \& Oechsle, 1990), (3) amount of exercise carried out by the respondent, (4) whether the respondents engaged in exercise or not, and (5) how much each participant's amount of exercise differed from the perceived average amount of exercise of other people. Both the perceived exercise rank and distance from the average amount of exercise variables were formed by (a) estimating each participant's cumulative distribution function (using either a lognormal or a linear function depending on best fit) from the answers they gave to the Section 3 "deciles" questions, and (b) calculating the mean of each participant's subjective distribution function and their own rank position within it. The findings from the first regression model are presented in Table 2, with unstandardized and standardized regression coefficients, $t$ values and probability statistics presented for each predictor variable.

In Step 1, the variables entered into the multiple regression significantly predicted perceived health benefits (adjusted $R^{2}=.27 ; F_{5,116}=9.96, p<.001$ ) with amount of exercise demonstrating a statistical significant regression coefficient. In Step 2, the inclusion of perceived exercise rank revealed a statistically significant 
Table 2 Two-Step multiple regression analysis with perceived health benefits relating to exercise as the dependent variable and sex, age, amount of exercise, whether the person exercises, and distance from the average exercise, entered as predictor variables in Step 1 and perceived exercise rank entered as predictor variable in Step 2

\begin{tabular}{lcccc}
\hline & B & $\boldsymbol{\beta}$ & $\boldsymbol{t}$ & $\boldsymbol{p}$ \\
\hline Step 1 & & & & \\
Sex & -.07 & -.01 & -.06 & .950 \\
Age & -.17 & -.03 & -.40 & .689 \\
Amount of Exercise & .03 & .54 & 6.35 & .001 \\
Exerciser or Nonexerciser & 5.01 & .11 & 1.43 & .156 \\
Distance from the Average Exercise & -.01 & -.08 & -1.00 & .321 \\
Step 2 & & & & \\
Sex & -.27 & -.02 & -.24 & .808 \\
Age & -.16 & -.03 & -.38 & .707 \\
Amount of Exercise & .02 & .30 & 2.05 & .042 \\
Exerciser or Nonexerciser & 4.11 & .09 & 1.18 & .241 \\
Distance from the Average Exercise & .01 & .08 & .71 & .482 \\
Perceived Exercise Rank & 6.19 & .29 & 2.05 & .043 \\
\hline
\end{tabular}

change $\left(R^{2}\right.$ change $=.025, F$ change $\left.{ }_{1,115}=4.18, p=.043\right)$ to the overall model (adjusted $R^{2}=.29 ; F_{6,115}=9.23, p<.001$ ), with amount of exercise and perceived exercise rank demonstrating statistical significant regression coefficients.

Two and a half percent of respondents reported not engaging in any exercise. These respondents may present a distinct group as nonexercisers may have insufficient information with which to judge their social rank with respect to exercise. Therefore we performed a second two-step hierarchical multiple regression with just those participants who reported exercising, excluding the variable that specified whether the individual exercised or not in Step 1. These findings followed a similar pattern to the one found in the first analysis, with the model for Step 1 reaching statistical significance (adjusted $R^{2}=.25 ; F_{4,114}=10.85, p<.001$ ) and a statistically significant regression coefficient being found for amount of exercise $(\beta=.54, t=$ $6.32, p<.001)$. The inclusion of perceived exercise rank in Step 2 again produced a statistically significant change $\left(R^{2}\right.$ change $=.027, F$ change $\left.{ }_{1,113}=4.32, p=.032\right)$ to the overall model (adjusted $R^{2}=.27 ; F_{5,113}=4.32, p<.001$ ), with amount of exercise $(\beta=.29, t=1.98, p=.049)$ and perceived exercise rank $(\beta=.30, t=2.08$, $p=.040$ ) demonstrating statistically significant regression coefficients. Thus selfperception of exercise rank predicts unique variance in the perceived health benefits of current exercise, after controlling for a number of relevant variables. The findings remain qualitatively unaltered if nonexercisers are excluded. 


\section{Study 2}

Study 2 tested whether manipulating the rank position of an amount (duration) of exercise causally influenced the participants' perceived health benefits of that exercise amount.

\section{Method}

Participants. One hundred thirty-five young adults (64 males; 71 females) aged between 18 and 22 years $(M=19.14 ; S D=0.95)$ completed a questionnaire. The method for recruitment was the same as described in Study 1 but took place in the Midlands area of England. Ethical approval for the study was obtained from the relevant university ethics committee, complying with the British Psychological Society Code of Ethics. Informed consent was obtained from all participants.

Procedure. Respondents were randomly assigned to one of two experimental groups and were each presented with 11 different amounts of exercise (as minutes per week). For each amount of exercise, participants were asked to make judgments about an individual who undertook that amount of exercise. They rated (1) how healthy it was for the individual (scored from 1 [Very Unhealthy] to 10 [Very Healthy]), (2) the extent of the health benefits (scored from 1 [No Benefit] to 10 [Huge Benefit]) and (3) how sufficient it would be to avoid the serious health problems associated with low levels of exercise (scored from 1 [Totally Insufficient] to 10 [Totally Sufficient]).

The quantities of amounts of exercise presented to respondents varied between the two experimental groups, forming the key experimental manipulation, and are illustrated in Table 3. The two groups were termed unimodal $(n=68)$ or bimodal ( $n=67)$ according to the distribution of quantities of amount of exercise.

The groups saw an equal number of amounts of exercise, ranging from 36 to 252 $\mathrm{min}$, and the average number of minutes in each distribution was $144 \mathrm{~min}$. Central to the experimental manipulation, the two groups had five exercise durations in common (36, 92, 144, 196 and $252 \mathrm{~min})$. If the participants simply rated each amount of exercise in isolation, or judged them according to distance from the mean, then the common amounts of exercise should be seen as having equal health benefits in both groups. However, if judgments were based on rank, then the common amounts of exercise should be judged as producing different levels of health benefits, depending on the experimental group. Specifically, where the common point is equal in rank in both groups (e.g., $36 \mathrm{~min}$ [rank 1], $144 \mathrm{~min}$ [rank 6] and $252 \mathrm{~min}$ [rank 11]) it should be seen as equally beneficial to health in each group. However, the common point of 92 min should be seen as less beneficial to health in the unimodal group (where its rank $=2$, i.e., the second lowest level) than in the bimodal group (where its rank =5). Similarly, if judgments are made in terms of relative ranked position, the common point of 196 min should be seen as more beneficial to health in the unimodal group (rank $=10$ ) than in the bimodal group (rank =7).

\section{Results}

As with Study 1, to examine whether the responses to the three perceived health benefits of exercise questions could be summed, we computed internal reliability and factorial validity estimates for the three questions across each common time point $(36,92,144,196$, and $252 \mathrm{~min})$ for both the unimodal and bimodal groups. 


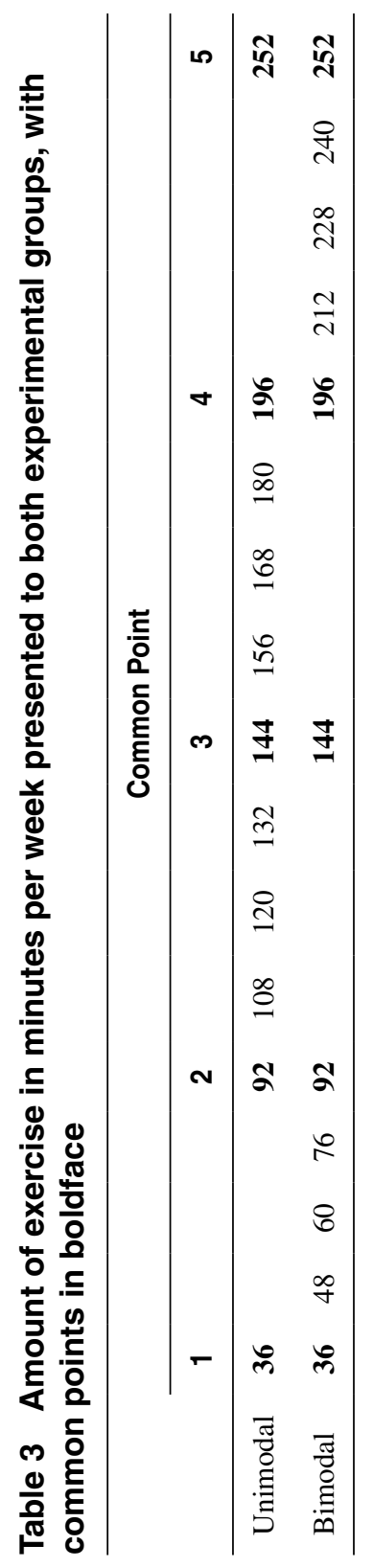


Across the two experimental groups and five common points, the Cronbach alpha (Cronbach, 1951) statistic ranged from $\alpha=.70$ to .86 . This is equal to or above the acceptable internal reliability criteria of .70. A maximum likelihood factor analysis was conducted to examine structural validity. A single factor (eigenvalues $>1.88$ ) explained at least $62.81 \%$ of the variance in each case. Potential second and third factors explained no more than $26.33 \%$ and $10.85 \%$ of the variance respectively, with all eigenvalues being below 1.00 , ranging from $<.79$, and $<.33$ respectively. Across the cases, item loadings on the unrotated first factor ranged from .42 to .99 . In addition, parallel analysis supported a one factor structure for each case, with the highest second eigenvalue (.79) failing to exceed the second of the three mean eigenvalues computed for each experimental group (1.19/1.19, $1.00 / .99$, and .81/.81). The results of these analyses suggest that the three questions can be combined into a composite score.

Mean ratings of perceived health benefits of exercise for the unimodal and bimodal groups across each of the five common points are presented in Table 4 . The interaction was tested using a 2 (between: group) $\times 5$ (within: common point) ANOVA. There was a main effect of comparison point $\left(F_{4,130}=321.89, p<.001, \eta_{\mathrm{p}}{ }^{2}=.908\right)$ and the interaction between the experimental group and the comparison point was significant $\left(F_{4,130}=164.08, p<.001, \eta_{\mathrm{p}}{ }^{2}=.84\right)$ suggesting that the perceived health benefits associated with exercising for a given number of minutes depended on the rank of that number of minutes within each condition. This interaction is illustrated in Figure 1a. Where the rank was the same in both groups at common points 1 (36 min, rank = 1), 3 (144 min, rank =6), and 5 (36 min, rank = 11), no significant differences were found between the unimodal and bimodal group in overall scores for the perceived health benefits of exercise (Common point $1, F=2.01, p=.158, \eta_{\mathrm{p}}{ }^{2}$ $=.02$; Common point $3, F=.69, p=.408, \eta_{\mathrm{p}}{ }^{2}=.01$; Common point $5, F=.49, p=$ $\left..486, \eta_{\mathrm{p}}{ }^{2}=.01\right)$. However, Common point $2(92 \mathrm{~min})$ was rated as significantly less beneficial to health $\left(F=68.98, p<.001, \eta_{\mathrm{p}}^{2}=.34\right)$ in the unimodal group (where rank $=2$ ) than in the bimodal group (where rank $=5$ ). Conversely, Common point 4 (196 min) was rated significantly more beneficial to health $\left(F=53.59, p<.001, \eta_{\mathrm{p}}{ }^{2}=.29\right)$ in the unimodal group (where rank $=10$ ) than in the bimodal group (where rank $=7$ ).

Table 4 Means and standard deviation scores for overall perceived
health benefits relating to exercise of individuals exercising for five
different "minutes of exercise" each week by unimodal and bimodal
groups

\begin{tabular}{lccccc}
\hline \multirow{2}{*}{$\begin{array}{l}\text { Common } \\
\text { Point }\end{array}$} & Minutes & $\begin{array}{c}\text { Unimodal } \\
\text { Rank }\end{array}$ & $\begin{array}{c}\text { Bimodal } \\
\text { Rank }\end{array}$ & \multicolumn{2}{c}{ Overall Perceived Health Benefits } \\
\cline { 6 - 7 } 1 & 36 & 1 st & 1 st & $8.90(4.07)$ & $7.94(3.75)$ \\
2 & 92 & 5 th & 2 nd & $10.66(4.61)$ & $17.33(4.71)$ \\
3 & 144 & 6 th & 6 th & $18.64(4.56)$ & $19.28(4.38)$ \\
4 & 196 & 7 th & 10 th & $25.37(4.39)$ & $19.87(4.34)$ \\
5 & 252 & 11 th & 11 th & $25.35(4.47)$ & $25.89(4.72)$ \\
\hline
\end{tabular}




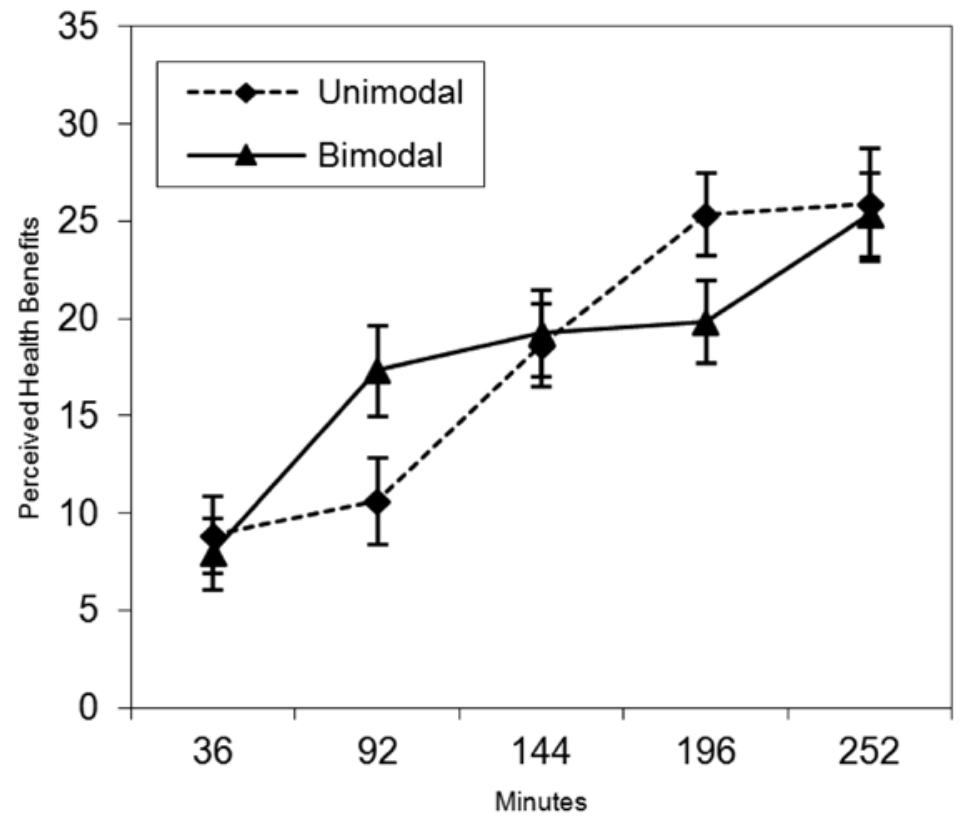

Figure 1 - Perceived health benefits of five different amounts of exercise as a function of experimental group.

\section{Discussion}

The results show that individuals' perceptions of the health benefits of exercise are influenced by how the amount of exercise ranks in comparison with the exercise amount of others. The exercise rank hypothesis was examined in two complementary ways. Study 1 demonstrated that individuals' perceptions of the health benefits of their own current exercise amounts were predicted by a rank-based judgment model, while controlling for sex, age, incidence, and prevalence of exercise, and distance of exercise from the mean. Study 2 demonstrated that perceptions of the health benefits of a given amount of exercise can be experimentally manipulated by changing the ranked position of the amount within a context. The findings provide empirical support for an exercise rank hypothesis as derived from an evolutionary explanation of motivation to produce physiologically healthy systems and the $\mathrm{DbS}$ model of cognitive judgments. Specifically, the findings suggest that individuals are both motivated and have the cognitive capacity to be influenced by social rank when assessing the health benefits of exercise. The current study provides an additional theoretical and empirical possibility for researchers to explore when examining social influences on exercise.

The findings have applied value and suggest that knowing the rank of one's own exercise level in relation to potential health benefits within a population may provide suitable encouragement for changing one's amount of exercise. Consequently, these findings have implications for intervention strategies and suggest 
that an effective implementation message could emphasize the rank positions of individuals, whether in addition or as an alternative to mean or average based information. In terms of being an additional source of information, the use of multiple information sources lends itself to "nudge-style" interventions that suggest that there are possibly multiple rational and automatic drivers to decision making (Thaler \& Sunstein, 2008; Dolan et al., 2012). Further consideration could be given to testing the relative efficacy of providing norms based on ranks or means/averages for changing exercise attitudes or behavior.

Further research is needed to examine whether these rank comparisons made around exercise are automatic comparative processes or reflective and deliberate cognitive decisions. The motivational and cognitive theoretical basis to the exercise rank hypothesis suggests that they represent natural and automatic comparisons, but the extent to which they act alone or may influence more reflective cognitions needs to be explored, not least so that any practical implications can be finely tuned.

Limitations of this study include a focus on exercise as a motivation for developing health systems, ignoring social psychological perspectives that could explore cultural differences across social groups, most notably where there may be differential emphasis on different health systems. Therefore an examination of how rank sensitivity and exercise judgments collate cross-culturally would be a useful extension of this research. Further research is needed to see whether the findings will generalize to other ages, perhaps most importantly younger populations where concerns around health development and the need to develop exercise habits are greatest (Currie, et al., 2006). Finally, the current study relies on self-report measures of exercise, and therefore further research might employ further assessments of exercise frequency, such as objective measures of physical activity such as pedometry, accelerometry, or heart rate monitoring (Corder, Ekelund, Steele, Wareham \& Brage, 2008).

In summary, findings from both studies show there is a statistically significant relationship between how an individual perceives how his or her amount of exercise ranks among other people and the perceived health benefits of their exercise. The results supports the exercise rank hypothesis, and introduces a theoretical and empirical approach for exploring how social ranks can influence perceptions of the health benefits of exercise.

\section{Acknowledgments}

The research was supported by grant RES-062-23-2462 from the Economic and Social Research Council (UK).

\section{References}

Ajzen, I. (1991). The theory of planned behavior. Organizational Behavior and Human Decision Processes, 50, 179-211. doi:10.1016/0749-5978(91)90020-T

Carron, A.V., Hausenblas, H.A., \& Mack, D.R. (1996). Social influence and exercise: A meta-analysis. Journal of Sport \& Exercise Psychology, 18, 1-16.

Centers for Disease Control and Prevention. (2011). Physical activity and health. (http:// www.cdc.gov/physicalactivity/everyone/health/index.html, accessed 8 April, 2012).

Cialdini, R.B., Kallgren, C.A., \& Reno, R.R. (1991). A focus theory of normative conduct: a theoretical refinement and re-evaluation of the role of norms in human behavior. 
Advances in Experimental Social Psychology, 24, 201-234. doi:10.1016/S00652601(08)60330-5

Corder, K., Ekelund, U., Steele, R.M., Wareham, N.J., \& Brage, S. (2008). Assessment of physical activity in youth. Journal of Applied Physiology, 105, 977-987. PubMed doi:10.1152/japplphysiol.00094.2008

Cronbach, L.J. (1951). Coefficient alpha and the internal structure of tests. Psychometrika, 16, 297-334. doi:10.1007/BF02310555

Cummins, D.D. (1996). Dominance hierarchies and the evolution of human reasoning. Minds and Machines, 6, 463-480.

Cummins, D.D. (2006). Dominance, status, and social hierarchies. In D.M. Buss (Ed.), The handbook of evolutionary psychology (pp. 676-697). Hoboken, NJ: Wiley.

Currie, C., . . ., eds. (2006). Young people's health in context. Health Behaviour in Schoolaged Children (HBSC) study: international report from the 2001/2002 survey. Copenhagen, WHO Regional Office for Europe, 2004 (Health Policy for Children and Adolescents, No. 4;http://www.euro.who.int/eprise/main/who/informationsources/ publications/catalogue/20040518_1, accessed 21 August 2006).

Deci, E.L., \& Ryan, R.M. (1985). Intrinsic motivation and self-determination in human behavior. New York: Plenum.

Dolan, P., Hallsworth, M., Halpern, D., King, D., Metcalfe, R., \& Vlaev, I. (2012). Influencing behaviour: The mindspace way. Journal of Economic Psychology, 33, 264-277. doi:10.1016/j.joep.2011.10.009

Eaton, S.B., Strassman, B.I., Nesse, R.M., Neel, J.V., Ewald, P.W., Williams, G.C., . . . Cordain, L. (2002). Evolutionary Health Promotion Preventive. Medicine, 34, 109-118.

Gibbons, F.X., Gerrard, M., Blanton, H., \& Russell, D.W. (1998). Cognative antecedents to adolescent health risk: Discriminating between behavioral intention and behavioral willingness. Psychology \& Health, 13, 319-339. doi:10.1080/08870449808406754

Kline, P. (1986). A handbook of test construction: Introduction to psychometric design. New York, NY: Methuen.

Melrose, K., Brown, G.D.A., \& Wood, A.M. (in press). Am I abnormal? Relative rank and social norm effects in judgments of anxiety and depression symptom severity. Journal of Behavioral Decision Making.

Messick, S. (1995). Validity of psychological assessment: Validation of inferences from persons' responses and performance as scientific inquiry into scoring meaning. The American Psychologist, 50, 741-749. doi:10.1037/0003-066X.50.9.741

Newton, T.L., Bane, C.M., Flores, A., \& Greenfield, J. (1999). Dominance, gender, and cardiovascular reactivity during social interaction. Psychophysiology, 36, 245-252. PubMed doi:10.1017/S0048577299971986

O’Keefe, J.H., Vogel, R., Lavie, C.J., \& Cordain, L. (2010). Organic fitness: Physical activity consistent with Our hunter-gatherer heritage. Physician and Sportmedicine, 38, 11-18. PubMed

O'Keefe, J.H., Vogel, R., Lavie, C.J., \& Cordain, L. (2011). Exercise like a hunter-gatherer: A prescription for organic physical fitness. Progress in Cardiovascular Diseases, 53, 471-479. PubMed doi:10.1016/j.pcad.2011.03.009

Ouellette, J.A., Hessling, R., Gibbons, F.X., Reis-Bergan, M., \& Gerrard, M. (2005). Using images to increase exercise behavior: Prototypes versus possible selves. Personality and Social Psychology Bulletin, 31, 610-620. PubMed doi:10.1177/0146167204271589

Parducci, A., \& Perrett, L.F. (1971). Category rating scales: Effects of relative spacing and frequency of stimulus values. Journal of Experimental Psychology Monograph, 89, 427-452. doi:10.1037/h0031258

Priebe, C.S., \& Spink, K.S. (2011). When in Rome: Descriptive norms and physical activity. Psychology of Sport and Exercise, 12, 93-98. doi:10.1016/j.psychsport.2010.09.001

Rhodes, R.E., Blanchard, C.M., Courneya, K.S., \& Plotnikoff, R.C. (2009). Identifying belief-based targets for the promotion of leisure-time walking. Health Education \& Behavior, 36, 381-393. PubMed doi:10.1177/1090198107308376 
Riskey, D.R., Parducci, A., \& Beauchamp, G.K. (1979). Effects of context in judgments of sweetness and pleasantness. Perception \& Psychophysics, 26, 171-176. doi:10.3758/ BF03199865

Sapolsky, R.M. (2004). Social status and health in humans and other animals. Annual Review of Anthropology, 33, 393-418. doi:10.1146/annurev.anthro.33.070203.144000

Stewart, N., Chater, N., \& Brown, G.D.A. (2006). Decision by sampling. Cognitive Psychology, 53, 1-26. PubMed doi:10.1016/j.cogpsych.2005.10.003

Thaler, R.H., \& Sunstein, C.R. (2008). Nudge: Improving decisions about health, wealth and happiness. London: Penguin.

Taylor, P.J., Gooding, P., Wood, A.M., \& Tarrier, N. (2011). The role of defeat and entrapment in depression, anxiety, and suicide. Psychological Bulletin, 137, 391-420. PubMed doi:10.1037/a0022935

Volden, C., Langemo, D., Adamson, M., \& Oechsle, L. (1990). The relationship of age, gender, and exercise practices to measure of health, life-style, and self-esteem. Applied Nursing Research, 3, 20-26. PubMed doi:10.1016/S0897-1897(05)80150-8

Wilson, E.O. (1975). Sociobiology: The new synthesis. Cambridge, MA: Belknap Press of Harvard University Press.

Wood, A.M., Brown, G.D.A., Maltby, J., \& Watkinson, P. (2012). How are personality judgments made? A cognitive model of reference group effects, personality scale responses, and behavioral reactions. Journal of Personality, 80, 1275-1311.

Wood, A.M., Brown, G.D.A., \& Maltby, J. (2012). Social norm influences on evaluations of the risks associated with alcohol consumption: Applying the rank-based decision by sampling model to health judgments. Alcohol and Alcoholism (Oxford, Oxfordshire), 47, 57-62. PubMed doi:10.1093/alcalc/agr146

World Health Organization. Geneva (2006). Diet and physical activity: A public health priority [web site]. (http://www.who.int/dietphysicalactivity/en, accessed 27th May 2011).

World Health Organization. Geneva (2004). Global strategy on diet, physical activity and health (http://www.who.int/dietphysicalactivity/strategy/eb11344/strategy_english_ web.pdf, Accessed $8^{\text {th }}$ April, 2012).

World Health Organization. (2010). Global strategy on diet, physical activity and health. Geneva. Geneva: World Health Organization.

Zwick, W.R., \& Velicer, W.F. (1986). Comparison of five rules for determining the number of components to retain. Psychological Bulletin, 99, 432-442. doi:10.1037/00332909.99.3.432

Manuscript submitted: January 9, 2012

Revision accepted: September 15, 2012 